\title{
Appendiceal Orifice Inflammation Mimicking Submucosal Tumor on Roentgenogram in Ulcerative Colitis
}

\author{
Mitsuro Chiba*, Tsuyotoshi Tsuji, Satoko Tsuda, Masafumi Komatsu \\ Division of Gastroenterology, Akita City Hospital, Akita, Japan \\ Email: ${ }^{*}$ mchiba@m2.gyao.ne.jp
}

Received 24 February 2014; revised 27 March 2014; accepted 6 April 2014

Copyright (C) 2014 by authors and Scientific Research Publishing Inc.

This work is licensed under the Creative Commons Attribution International License (CC BY). http://creativecommons.org/licenses/by/4.0/

c) (7) Open Access

\begin{abstract}
Appendiceal orifice inflammation (AOI) is observed as skip lesion in distal ulcerative colitis (UC). The endoscopic frequency of $\mathrm{AOI}$ is reported to be $7.9 \%$ to $76 \%$ of distal colitis. UC is a relapsing and remitting disease and a morphological change of the large bowel occurs over time. Patient and physician can easily understand the shape of the whole large bowel not through endoscopic photograph but by roentgenogram of the bowel. Therefore, the authors undertake barium enema study when a diagnosis of UC is made. We have experienced a patient with proctitis in which an appendiceal submucosal tumor (SMT) was suspected on the roentgenogram but it was turned out to be a protruding lesion of AOI. A 16-year-old boy visited us with complaining of blood in his stool for the past 10 days. Sigmoidoscopy revealed mild diffuse inflammation. Crypt abscess was found in biopsy specimen. A diagnosis of UC, proctitis type, was made. A double contrast barium enema study revealed a defect shadow with a smooth surface, length $37 \mathrm{~mm}$, height $12 \mathrm{~mm}$, over the appendix. An appendiceal SMT was suspected. Abdominal ultrasonography and computed tomography were non-contributory. Colonoscopy revealed a spiral inflamed mucosa at the site of an appendiceal orifice. There was an inflammation in the cecum surrounding the orifice. Biopsy specimen of the appendiceal orifice showed inflammatory cells infiltration, goblet cell depletion, and cryptitis. The suspected SMT lesion was concluded to be AOI.
\end{abstract}

\section{Keywords}

Appendix, Ulcerative Colitis, Submucosal Tumor

\footnotetext{
*Corresponding author.
} 


\section{Introduction}

Appendiceal orifice inflammation (AOI) is observed as skip lesion in distal ulcerative colitis (UC) [1]-[4]. Three forms of AOI are described: inflammation is limited to the site of appendiceal orifice, inflammation extending to an area surrounding the orifice, and inflammation extending to the cecum [3]. The endoscopic frequency of AOI is reported to be $7.9 \%$ to $76 \%$ of distal colitis [1]-[4]. UC is a relapsing and remitting disease and a morphological change of the large bowel occurs over time. Patient and physician can easily understand the shape of the whole large bowel not through endoscopic photograph but by roentgenogram of the bowel. Therefore, the authors undertake barium enema study when a diagnosis of UC is made. We have experienced a patient with proctitis in which roentgenogram indicated an appendiceal submucosal tumor (SMT) but it turned out to be a protruding lesion of AOI. Such a case has not been reported before.

\section{Case Report}

On February 16, 2009, a 16-year-old boy visited us with complaining of blood in his stool for the past 10 days. He had bronchial asthma until 6-year-old. Sigmoidoscopy revealed mild diffuse inflammation in the rectum. Crypt abscess was found in his biopsy specimen. Stool culture was negative for pathogens. A diagnosis of UC, proctitis type, was made. Since the symptom was mild, the responsible doctor (MC) proposed to him an educational admission of a short period during his spring vacation. Until admission he was advised to have prudent meals and lead a normal life. He was admitted on March 23, when bloody stool was absent. A routine examination of his blood was normal. Fecal occult blood tests were $213 \mathrm{ng} / \mathrm{ml}$ and $140 \mathrm{ng} / \mathrm{ml}$ (normal range $<100 \mathrm{ng} / \mathrm{ml}$ ). He was provided $2000 \mathrm{kcal} / \mathrm{d}$ of a semi-vegetarian diet (SVD) [5]. A double contrast barium enema study on March 26 was non-contributory except for a defect shadow with smooth surface, length $37 \mathrm{~mm}$ : height $12 \mathrm{~mm}$, over the appendix (Figure 1). An appendiceal SMT was suspected. Abdominal ultrasonography and computed tomography were non-contributory. Colonoscopy on March 31 revealed an inflamed, edematous mucosa at the site of appendiceal orifice and mild inflammation surrounding the orifice (Figure 2(A)). The orifice was protruded and it was found out to be a spiral inflamed mucosa by forceps manipulation (Figure 2(B)). Biopsy specimen of the orifice showed inflammatory cells infiltration, goblet cell depletion, and cryptitis. The suspected SMT lesion was concluded to be AOI.

On April the $1^{\text {st }}$, he was found to have a low grade fever at noon. Therefore, mesalazine (Pentasa), $1.5 \mathrm{~g} / \mathrm{d}$, was started, and the fever disappeared. The patient and his mother were provided a dietary guidance of SVD [5]. He was discharged on April 4 (13 days of hospitalization).

Eight months later, after eight months remission, colonoscopy was repeated. The rectum showed a picture of remission. Mild inflammation in the cecum surrounding the orifice disappeared. But protruded spiral inflamed
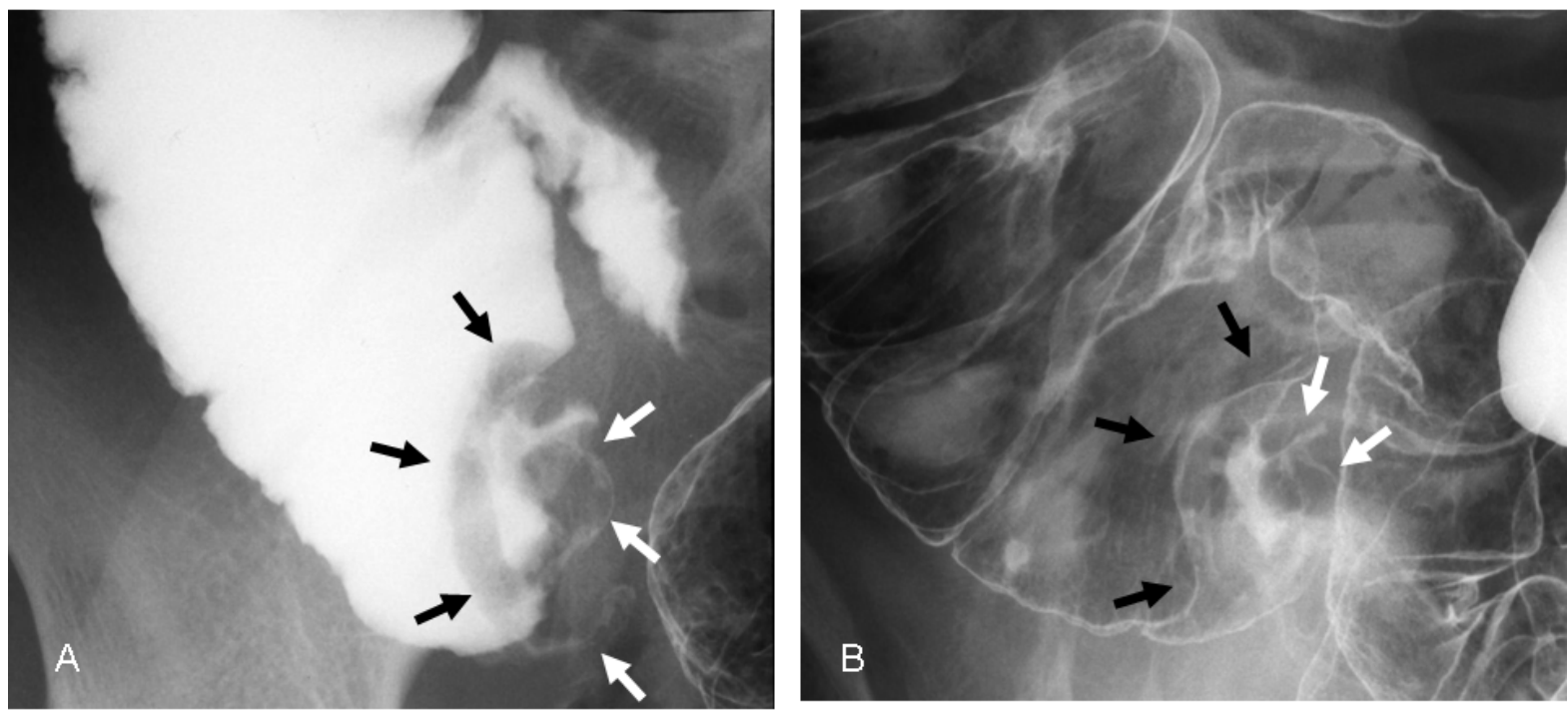

Figure 1. Roentgenogram of a double contrast barium enema study. A defect shadow indicated by black arrows with smooth surface, length $37 \mathrm{~mm}$ : height $12 \mathrm{~mm}$, was seen over the appendix ((A), (B)). The appendix is indicated by white arrows. 

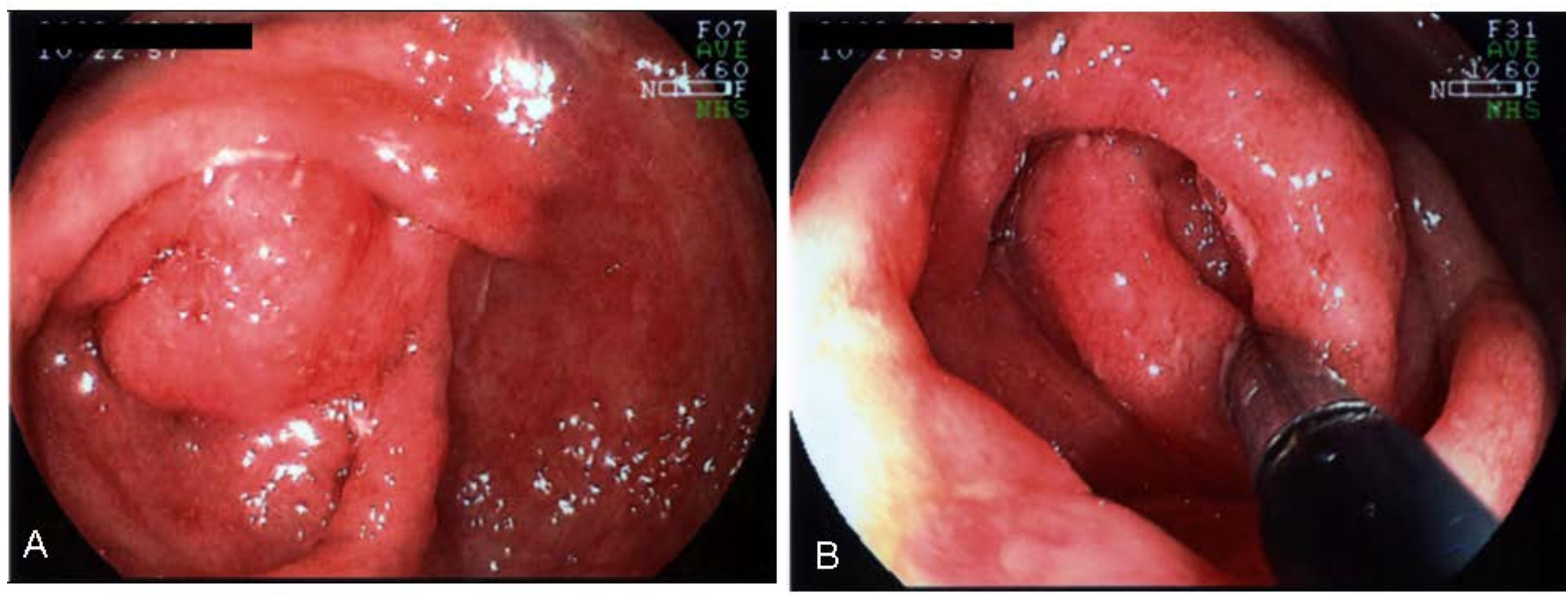

Figure 2. Endoscopic picture of colonoscopy. A protruded, inflamed, edematous mucosa was seen at the site of appendiceal orifice and mild inflammation surrounding the orifice (A). The protruded lesion was found out to be a spiral inflamed mucosa by forceps manipulation (B).

mucosa was evident as before. He had been well up to the present, December 2013, although he had occasional bloody stool which did not require hospitalization or change of medication.

\section{Discussion}

Inflammatory bowel disease is a lifestyle-related disease mediated mainly with westernized diet [5] [6]. Therefore, diet is crucial to maintain remission. SVD is found to be effective for preventing relapse in CD [5]. Diet in Japan has been westernized and is now far from SVD. Therefore, experience of and familiarization with SVD is needed. This can be attained by hospitalization for a short period of around 2 weeks. During such hospitalization, the present case was found to have AOI and it looked like SMT on the roentgenogram. After this experience, we paid attention to an endoscopic form of AOI. We recognize that the flat type of AOI is most common but a protruding type of AOI, like the present case, is occasionally observed (unpublished observation).

\section{Conclusion}

There is a protruding type of AOI, although it is rare, and it looks like SMT on roentgenogram.

\section{References}

[1] D’Haens, G., Geboes, K., Peeters, M., Baert, F., Ectors, N. and Rutgeerts, P. (1997) Patchy Cecal Inflammation Associated with Distal Ulcerative Colitis: A Prospective Endoscopic Study. American Journal of Gastroenterology, 92, 1275-1279.

[2] Yang, S.K., Jung, H.Y., Kang, G.H., et al. (1999) Appendiceal Orifice Inflammation as a Skip Lesion in Ulcerative Colitis: An Analysis in Relation to Medical Therapy and Disease Extent. Gastrointestinal Endoscopy, 49, $743-747$. http://dx.doi.org/10.1016/S0016-5107(99)70293-2

[3] Matsumoto, T., Nakamura, S., Shimizu, M. and Iida, M. (2002) Significance of Appendiceal Involvement in Patients with Ulcerative Colitis. Gastrointestinal Endoscopy, 55, 180-185. http://dx.doi.org/10.1067/mge.2002.121335

[4] Rubin, D.T. and Rothe, J.A. (2010) The Peri-Appendiceal Red Patch in Ulcerative Colitis: Review of the University of Chicago Experience. Digestive Diseases and Sciences, 55, 3495-3501. http://dx.doi.org/10.1007/s10620-010-1424-X

[5] Chiba, M., Abe, T., Tsuda, H., et al. (2010) Lifestyle-Related Disease in Crohn's Disease: Relapse Prevention by a Semi-Vegetarian Diet. World Journal of Gastroenterology, 16, 2484-2495. http://dx.doi.org/10.3748/wjg.v16.i20.2484

[6] Chiba, M., Tsuda, H., Abe, T., Sugawara, T. and Morikawa, Y. (2011) Missing Environmental Factor in Inflammatory Bowel Disease: Diet-Associated Gut Microflora. Inflammatory Bowel Diseases, 17, E82-E83. http://dx.doi.org/10.1002/ibd.21745 